Provided for non-commercial research and education use. Not for reproduction, distribution or commercial use.

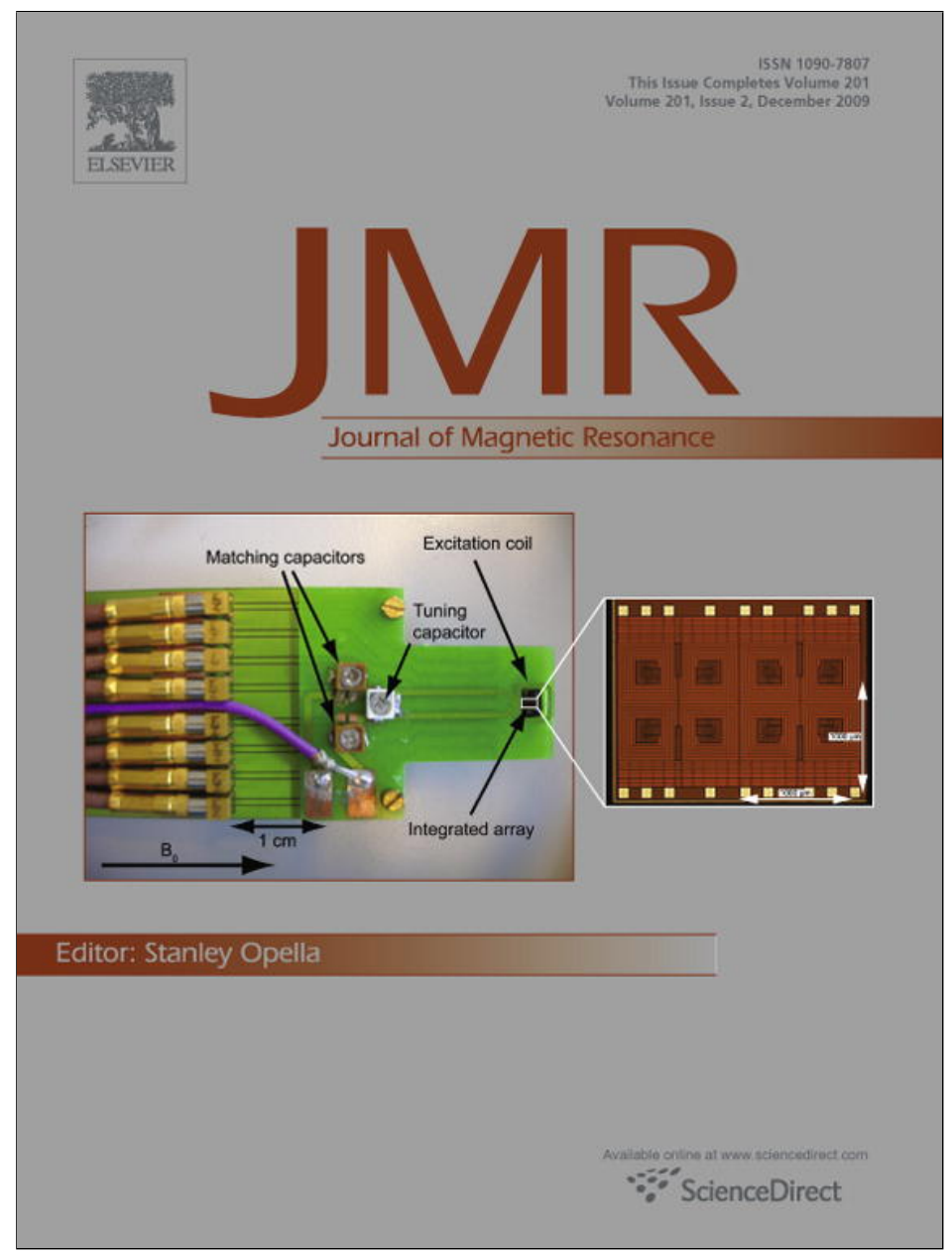

This article appeared in a journal published by Elsevier. The attached copy is furnished to the author for internal non-commercial research and education use, including for instruction at the authors institution and sharing with colleagues.

Other uses, including reproduction and distribution, or selling or licensing copies, or posting to personal, institutional or third party websites are prohibited.

In most cases authors are permitted to post their version of the article (e.g. in Word or Tex form) to their personal website or institutional repository. Authors requiring further information regarding Elsevier's archiving and manuscript policies are encouraged to visit:

http://www.elsevier.com/copyright 


\title{
Cross-encoded magnetic resonance imaging in inhomogeneous fields
}

\author{
Raphael Paquin $^{\mathrm{a}, \mathrm{b}, *}$, Philippe Pelupessy ${ }^{\mathrm{a}, *}$, Geoffrey Bodenhausen ${ }^{\mathrm{a}, \mathrm{b}}$ \\ ${ }^{a}$ Département de Chimie, associé au CNRS, Ecole Normale Supérieure, 24 rue Lhomond, 75231 Paris Cedex 05, France \\ ${ }^{\mathrm{b}}$ Institut des Sciences et Ingénierie Chimiques, Ecole Polytechnique Fédérale de Lausanne, Batochime, 1015 Lausanne, Switzerland
}

\section{A R T I C L E I N F O}

\section{Article history:}

Received 29 July 2009

Revised 28 August 2009

Available online 11 September 2009

\section{Keywords:}

Magnetic resonance imaging (MRI)

Echo-planar imaging (EPI)

Inhomogeneous magnetic fields

Adiabatic radio-frequency pulses

Susceptibility effects

Slow motion

\begin{abstract}
A B S T R A C T
In magnetic resonance imaging (MRI), it is possible to cancel the effects of severe inhomogeneities of the magnetic field even if the field profile is unknown. The new 'cross-encoded' method is based on adiabatic frequency-modulated pulses combined with two orthogonal gradients that are applied simultaneously during encoding and decoding. Undistorted two- and three-dimensional images can be obtained in inhomogeneous fields where the breadth of the water resonance extends over several $\mathrm{kHz}$.
\end{abstract}

(c) 2009 Elsevier Inc. All rights reserved.

\section{Introduction}

Magnetic resonance imaging (MRI) is undoubtedly the most popular application of nuclear magnetic resonance [1], with wide-ranging implications for medical diagnosis, neurosciences, metabolism, and material science. Although the objects or living organisms should ideally be immersed in a magnetic field that is as homogeneous as possible, it is often too difficult or costly to fulfill this stringent requirement. In living organisms, the quality of the images is degraded in the vicinity of voids and surgical implants. Inanimate objects may also suffer from discontinuities of the magnetic susceptibility. Temporal variations that occur in stimulated brains mapped by functional MRI [2,3] call for fast imaging techniques, such as echo planar imaging (EPI) introduced by Mansfield and co-workers [4]. Inspired by these experiments, Frydman and co-workers introduced single-scan methods for 'ultrafast' spectroscopy [5]. Recently, these ideas were extended to imaging [6], making it possible to probe 'real' Cartesian space directly without relying on Fourier transformation [7] or back-projection [1]. These methods were designed to cope with inhomogeneous fields with known profiles, where the encoding schemes can be tailored to compensate for the phase dispersion arising from the inhomogeneity of the static field [8]. In this work, we shall present cross-encoded single-scan methods that are designed to compensate for effects of inhomogeneous fields in a frac-

\footnotetext{
* Corresponding authors.

E-mail addresses: raphael.paquin@ens.fr (R. Paquin), philippe.pelupessy@ens.fr (P. Pelupessy).
}

tion of a second without requiring any knowledge of their spatial profiles.

\section{Principles}

\subsection{Homogeneous fields}

In Fig. 1, a selective pulse combined with a gradient $G_{y}^{s}$ is applied along the $y$ axis to excite the magnetization in a $\{x, z\}$ plane. In contrast to earlier methods for single-scan spectroscopy [9], orthogonal $G_{x}^{e}$ encoding gradients of duration $T_{e}$ must be combined with $G_{z}^{e}$ encoding gradients. In the decoding interval, the $G_{z}^{d}$ gradients unwind the phase accumulated during the $G_{x}^{e}$ encoding gradients, instead of the chemical shifts in our earlier methods. We assume that only a single chemical shift $\Omega=0$ is present. Thus the precession frequency of the magnetization associated with a voxel centered on the coordinates $\vec{r}=\{x, y, z\}$ during the first adiabatic pulse is

$\omega_{\vec{r}}=\gamma \vec{G} \cdot \vec{r}=\gamma\left(G_{x}^{e} x+G_{z}^{e} z\right)$

The sign of $G_{z}^{e}$ is opposite during the second adiabatic pulse. The frequency sweep of the adiabatic pulse $-\Delta \omega_{a d} / 2 \leqslant \omega_{R F} \leqslant \Delta \omega_{a d} / 2$ is defined so that the center of the sweep corresponds to the Larmor frequency in the absence of any gradients. The phase at the end of the encoding block is:

$\varphi_{e}(x, z)=-\lambda x z$

where 

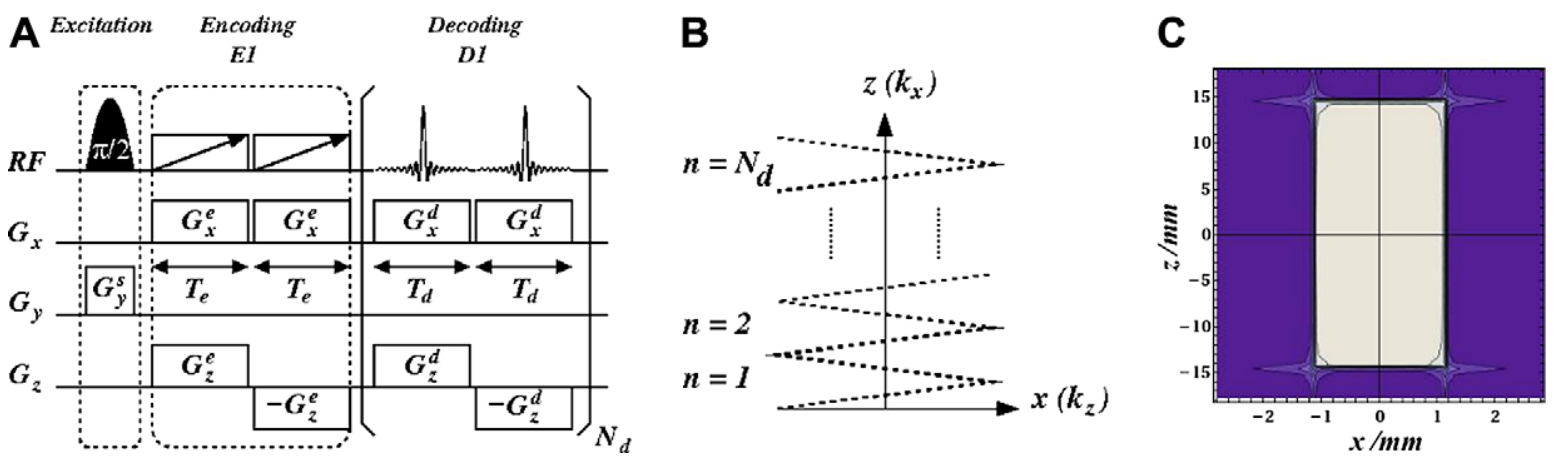

Fig. 1. Characteristics of cross-encoded magnetic resonance imaging (CE-MRI). (A) Excitation of transverse magnetization in a slice $\{x, z\}$ perpendicular to the $y$ axis is achieved with a selective radio-frequency $(R F)$ pulse in the presence of a field gradient $G_{y}^{s}$. The simplest encoding sequence $E 1$ comprises two adiabatic $R F$ pulses (rectangles with diagonal arrows) of duration $T_{e}$, applied in conjunction with a pair of opposite gradients $G_{z}^{e}$ and a pair of identical gradients $G_{x}^{e}$. In the simplest case, decoding $D 1$ is achieved by applying two identical gradients $G_{x}^{d}$ and a pair of opposite gradients $G_{z}^{d}$, each of duration $T_{d}$. This scheme is repeated $N_{d}$ times. (B) The Cartesian (real) $\{x, z\}$ space is probed directly through zig-zag trajectories imposed by the decoding sequence. (C) Numerical simulation of the image of a rectangular two-dimensional object of dimensions $2 \times 30 \mathrm{~mm}$, obtained with the pulse scheme (A). The absolute value of the integral of Eq. (4) has been calculated at intervals $\Delta x=x_{\max } / 100$ and $\Delta z=z_{\mathrm{max}} / 100 \mathrm{for}-x_{\max } / 2<x<$ $x_{\max } / 2$ and $-z_{\max } / 2<x<z_{\max } / 2$ with the following parameters $\gamma\left({ }^{1} \mathrm{H}\right)=2.6752 \mathrm{rad} /(\mathrm{T} \mathrm{s}), G_{x}^{e}=G_{z}^{e}=G_{x}^{d}=0.022 \mathrm{~T} / \mathrm{m}, G_{z}^{d}=0.22 \mathrm{~T} / \mathrm{m}, T_{e}=6 \mathrm{~ms}, T_{d}=320 \mu \mathrm{s}, \Delta \omega_{a d} /(2 \pi)=40 \mathrm{kHz}$, $N_{d}=32$, resulting in $x_{\max } \sim 5.7 \mathrm{~mm}$ and $z_{\max } \sim 36.4 \mathrm{~mm}$.

$$
\lambda=4 T_{e} \gamma^{2} G_{x}^{e} G_{z}^{e} / \Delta \omega_{a d}
$$

This equation is symmetric with respect to a permutation of the $x$ and $z$ coordinates. Thus, if the $G_{z}^{e}$ gradient encodes for the $x$ coordinate, the $G_{x}^{e}$ gradient will do the same for the $z$ coordinate. In contrast to traditional $k$-space MRI using Fourier transformations, the decoding sequence leads to a trajectory in the $\{x, z\}$ plane [8], which can be chosen at will. Fig. 1A shows a decoding scheme $D 1$ based on the simultaneous application of gradients $G_{x}^{d}$ and $G_{z}^{d}$ of duration $T_{d}$. Pairs of such gradients, with alternating signs in the latter dimension, must be repeated $N_{d}$ times. This procedure gives the 'zig-zag' trajectory illustrated in Fig. 1B [8]. The signal at time $t$ is

$$
S(t)=\iint_{\text {allx,z }} A(x, z) \exp \left[i \varphi_{e}(x, z)\right] \times \exp \left[i k_{x}(t) x+i k_{z}(t) z\right] d x d z
$$

where $A(x, z)$ is the amplitude of the initial longitudinal magnetization, and $k_{x}(t)$ and $k_{z}(t)$ are defined as

$$
\begin{aligned}
& k_{x}(t)=\gamma \int_{0}^{t} G_{x}^{d}\left(t^{\prime}\right) d t^{\prime} \quad k_{z}(t)=\gamma \int_{0}^{t} G_{z}^{d}\left(t^{\prime}\right) d t^{\prime} \\
& G_{x}^{d}\left(t^{\prime}\right)=G_{x}^{d} \quad G_{z}^{d}\left(t^{\prime}\right)=(-1)^{n_{D 1}\left(t^{\prime}\right)} G_{z}^{d}
\end{aligned}
$$

where $n_{D 1}\left(t^{\prime}\right)$ is the integer part of the fraction $t^{\prime} / T_{d}$, which toggles between +1 or -1 for positive and negative lobes of the train of bipolar decoding gradients.

As explained by Shrot and Frydman [6], the overall phase $\Phi$ should not vary across a voxel $V_{k}$ centered on the coordinates $\left(x_{k}, z_{k}\right)$

$\frac{\partial}{\partial x}[\Phi(x, z, t)]_{\left(x_{k}, z_{k}\right)}=0$ and $\frac{\partial}{\partial z}[\Phi(x, z, t)]_{\left(x_{k}, z_{k}\right)}=0$

with

$\Phi(x, z, t)=\varphi_{e}(x, z)+k_{x}(t) x+k_{z}(t) z$

From Eqs. (2), (7), and (8), the relation between the coordinates $\left(x_{k}, z_{k}\right)$ of the voxel $V_{k}$ and the variables $k_{x}(t)$ and $k_{z}(t)$ is:

$x_{k}=\frac{k_{z}\left(t_{k}\right)}{\lambda}$ and $z_{k}=\frac{k_{x}\left(t_{k}\right)}{\lambda}$

Thus at time $t_{k}$ in the decoding sequence, an echo signal appears that reveals the position $\left(x_{k}, z_{k}\right)$ of the voxel $V_{k}$. The $x$ dimension is decoded by the $z$ gradient and vice versa. While the $k_{x}$ "walk" oc- curs during $2 N_{d} T_{d}$ due to the continuous application of $G_{x}^{d}$, the $k_{z}$ trajectory is reversed every $T_{d}$ when the sign of $G_{z}^{d}$ changes.

In Fig. $1 \mathrm{C}$, an image that could be obtained with the adiabatic pulse scheme of Fig. $1 \mathrm{~A}$ is simulated for an ideal rectangular object. Only minor distortions are apparent around the edges of the object, in particular near the corners.

\subsection{Inhomogeneous fields}

In a static (time-independent) inhomogeneous field, the precession frequency of the magnetization of a voxel $V_{k}$ in the encoding interval is $\omega_{\vec{r}}=\gamma\left\{G_{x}^{e} x+G_{z}^{e} z+\delta B_{0}(x, y, z)\right\}$. The additional term is due to the (unknown) spatial variation of the static field $B_{0}(x, y$, $z)$. The phase at the end of the simple encoding scheme $E 1$ of Fig. 1 is:

$\varphi_{E 1}(x, z)=-\lambda_{E 1} x z$

where

$\lambda_{E 1}=4 T_{e} \gamma^{2}\left(G_{x}^{e} G_{z}^{e}+G_{z}^{e} \delta B_{0}(x, y, z) / x\right) / \Delta \omega_{a d}$

Compensation of static inhomogeneities during encoding can be achieved by the scheme of Fig. 2. After the initial encoding sequence $E 1$, a second sequence $E 2$ is inserted, where the signs of

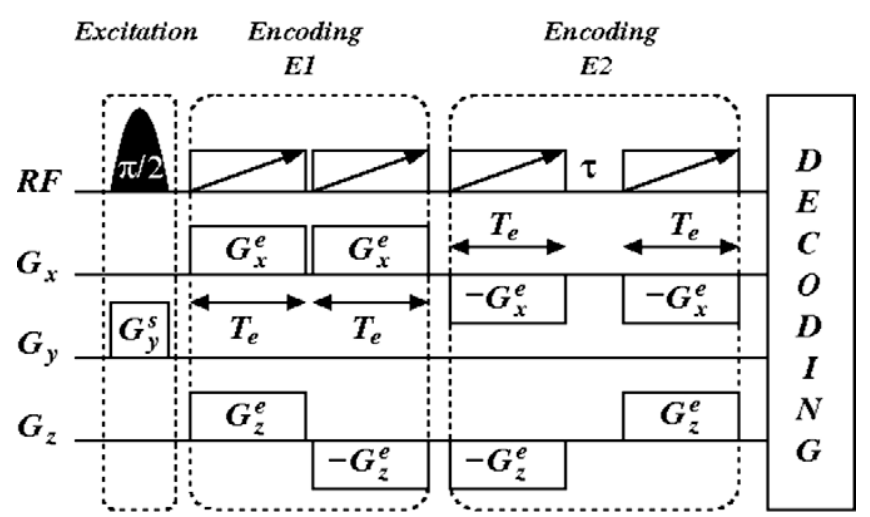

Fig. 2. Cross-encoded scheme designed to compensate for unknown inhomogeneities of the main field. In the second encoding block E2, the signs of all gradients are inverted with respect to $E 1$. The combined encoding blocks $(E 1+E 2)$ yield a phase of the transverse magnetization where the effects of all inhomogeneities are cancelled. A delay $\tau$ can be inserted between the two adiabatic pulses in $E 2$ to attenuate the distortions of the trajectories during the decoding sequence. 
all gradients are inverted. If the interval $\tau=0$ in $E 2$, the phase resulting from the encoding unit $E 2$ alone is

$\varphi_{E 2}(x, z)=-\lambda_{E 2} x z$

with

$\lambda_{E 2}=4 T_{e} \gamma^{2}\left(G_{x}^{e} G_{z}^{e}-G_{z}^{e} \delta B_{0}(x, y, z) / x\right) / \Delta \omega_{a d}$

Consequently, the effects of inhomogeneous static fields are cancelled by concatenating sequences $E 1$ and $E 2$ :

$\varphi_{e}=-2 \lambda x z$

where $\lambda$ is defined in Eq. (3).

We now focus our attention on the decoding sequence. If we combine the encoding schemes $E 1+E 2$ of Fig. 2 with the decoding part $D 1$ of Fig. $1 \mathrm{~A}$, the spatial variation of the static field $\delta B_{0}(x, y, z)$ yields an additional contribution to the overall phase

$\Phi(x, y, z, t)=\varphi_{e}(x, z)+k_{x}(t) x+k_{z}(t) z+\gamma \delta B_{0}(x, y, z) t$

This phase must again fulfill the conditions of Eq. (7):

$x_{k}-\frac{\gamma t_{k}}{2 \lambda}\left\{\frac{\partial \delta B_{0}(x, y, z)}{\partial z}\right\}_{\left(x_{k}, y_{k}, z_{k}\right)}=\frac{k_{z}\left(t_{k}\right)}{2 \lambda}$

$z_{k}-\frac{\gamma t_{k}}{2 \lambda}\left\{\frac{\partial \delta B_{0}(x, y, z)}{\partial x}\right\}_{\left(x_{k}, y_{k}, z_{k}\right)}=\frac{k_{x}\left(t_{k}\right)}{2 \lambda}$

The zig-zag trajectory now deviates from the ideal behavior in a homogeneous field because of the terms that are proportional to $t_{k}$ and to the derivatives of $\delta B_{0}(x, y, z)$. The signal from the voxel $V_{k}$ will be affected by static inhomogeneities if the time $t_{k}$ at which the echo appears is delayed.

During decoding, we can attenuate the effects of static field inhomogeneities but not suppress them completely. A first approximate solution (scheme $D 2$ in Fig. $3 \mathrm{~A}$ ) relies on the insertion of a composite pulse $(\pi / 2)_{y}(\pi)_{x}(\pi / 2)_{y}[10]$ between each pair of decoding gradients as can be done in EPI experiments $[11,12]$. This leads to an overall phase

$\Phi(x, y, z, t)=\varphi_{e}(x, z)+k_{x}(t) x+k_{z}(t) z+\gamma \delta B_{0}(x, y, z) f_{D 2}(t)$

The sign of the term $\gamma \delta B_{0}(x, y, z) f_{D 2}(t)$ is reversed at $t=(2 n-1) T_{d}$ with $n=1,2, \ldots, N_{d}$. In analogy to $k_{z}(t)$ in Eqs. (5) and (6), we define:

$f_{D 2}(t)=\int_{0}^{t}(-1)^{n_{D 2}\left(t^{\prime}\right)} d t^{\prime}$

where $n_{D 2}\left(t^{\prime}\right)$ is the integer part of $t^{\prime} /(2 T d)+1 / 2$, resulting in:

$x_{k}-\frac{\gamma f_{D 2}\left(t_{k}\right)}{2 \lambda}\left\{\frac{\partial \delta B_{0}(x, y, z)}{\partial z}\right\}_{\left(x_{k}, y_{k}, z_{k}\right)}=\frac{k_{z}\left(t_{k}\right)}{2 \lambda}$
$z_{k}-\frac{\gamma f_{D 2}\left(t_{k}\right)}{2 \lambda}\left\{\frac{\partial \delta B_{0}(x, y, z)}{\partial x}\right\}_{\left(x_{k}, y_{k}, z_{k}\right)}=\frac{k_{x}\left(t_{k}\right)}{2 \lambda}$

The most severe error in the localization of the voxel of interest $V_{k}$ occurs when $t_{k}=(2 n-1) T_{d}$
A

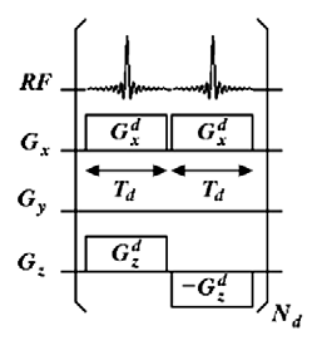

B

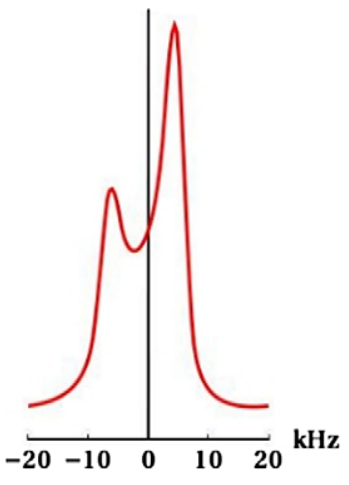

D2

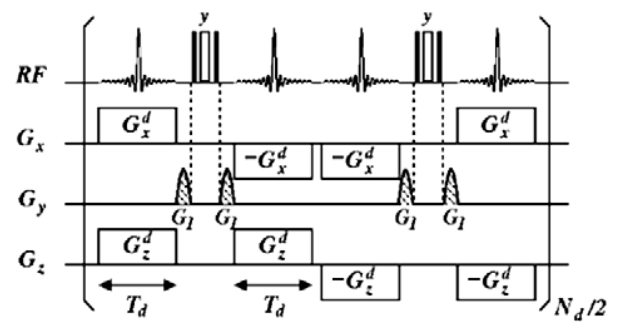

D1

$(\tau=0)$

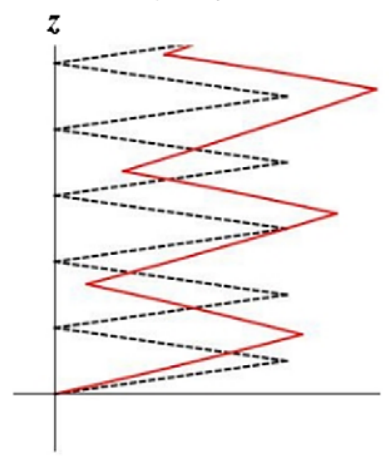

D2

$(\tau=0)$

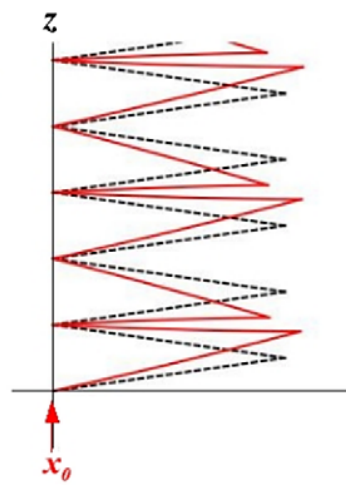

D3

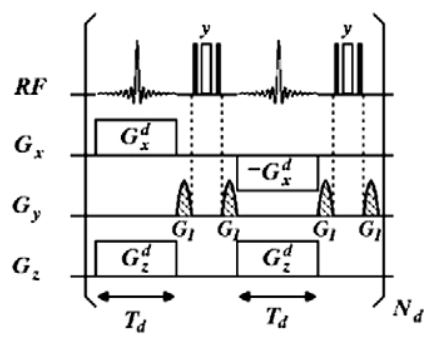

D3

$\left(\tau=T_{d} / 2\right)$

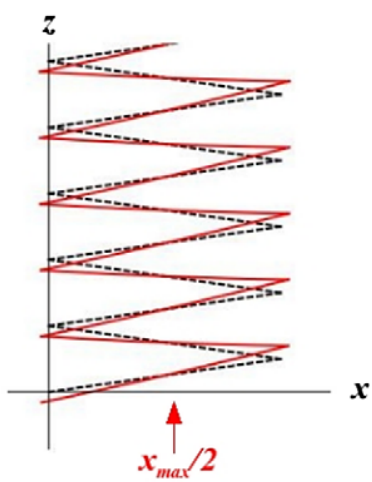

Fig. 3. (A) Comparison of the basic decoding scheme $D 1$ of Fig. $1 \mathrm{~A}$ with two alternative methods $D 2$ and $D 3$. In scheme $D 2$, composite refocusing pulses $90^{\circ}{ }_{x} 180^{\circ}{ }_{y} 90^{\circ}{ }_{x}$ flanked by two gradients $G_{1}$ are inserted between each pair of decoding gradients, while in scheme $D 3$ they are inserted after each gradient. (B) Simulated proton spectra and zig-zag trajectories in homogeneous (black dashed lines) and inhomogeneous fields (red continuous lines). The proton spectrum arising from a $4 \times 4 \times 20$ mm sample shows a fullwidth at half maximum of $\sim 20 \mathrm{kHz}$. Scheme $D 2$ entirely refocuses deviations from the ideal trajectory at $t=2(n-1) T_{d}$ with $n=1,2, \ldots, N_{d}$ but leaves errors at $t=(2 n-1) T_{d}$. When a delay $\tau=T_{d} / 2$ is inserted in the encoding sequence $E 2$ of Fig. 2, scheme $D 3$ results in distorted and ideal trajectories that intersect halfway at $x_{\text {max }} / 2$ rather than at $x_{0}$, and the deviations are reduced by a factor two. (For interpretation of the references to color in this figure legend, the reader is referred to the web version of this paper.) 


$$
\begin{aligned}
& \Delta x_{k}=\frac{\gamma T_{d}}{2 \lambda}\left\{\frac{\partial \delta B_{0}(x, y, z)}{\partial z}\right\}_{\left(x_{k}, y_{k}, z_{k}\right)} \\
& \Delta z_{k}=\frac{\gamma T_{d}}{2 \lambda}\left\{\frac{\partial \delta B_{0}(x, y, z)}{\partial x}\right\}_{\left(x_{k}, y_{k}, z_{k}\right)}
\end{aligned}
$$

In the alternative scheme $D 3$ in Fig. 3A, a composite pulse $(\pi / 2)_{y}(\pi)_{x}(\pi / 2)_{y}$ is inserted after each decoding gradient, and a delay $\tau=T_{d} / 2$ is used in E2 (see Fig. 2). The overall phase becomes

$$
\begin{aligned}
\Phi(x, y, z, t)= & \varphi_{e}(x, z)+k_{x}(t) x+k_{z}(t) z+\gamma \delta B_{0}(x, y, z) f_{D 3}(t) \\
& -\gamma \delta B_{0}(x, y, z) \frac{T_{d}}{2}
\end{aligned}
$$

where

$f_{D 3}(t)=\int_{0}^{t}(-1)^{n_{D 3}\left(t^{\prime}\right)} d t^{\prime}$

with $n_{D 3}\left(t^{\prime}\right)=n_{D 1}\left(t^{\prime}\right)$ so that

$x_{k}-\frac{\gamma\left(f_{D 3}\left(t_{k}\right)-T_{d} / 2\right)}{2 \lambda}\left\{\frac{\partial \delta B_{0}(x, y, z)}{\partial z}\right\}_{\left(x_{k}, y_{k}, z_{k}\right)}=\frac{k_{z}\left(t_{k}\right)}{2 \lambda}$

$z_{k}-\frac{\gamma\left(f_{D 3}\left(t_{k}\right)-T_{d} / 2\right)}{2 \lambda}\left\{\frac{\partial \delta B_{0}(x, y, z)}{\partial x}\right\}_{\left(x_{k}, y_{k}, z_{k}\right)}=\frac{k_{x}\left(t_{k}\right)}{2 \lambda}$

The error in the localization of the voxel $V_{k}$ is halved compared to Eq. (20). This error reaches a maximum at intervals $t_{k}=T_{d}$.

In Fig. 3B, we simulate the zig-zag trajectories for the three schemes $D 1, D 2$ and $D 3$, describing the intrinsic (time-independent) inhomogeneity of the static field by second-order polynomials:

$\delta B_{0}(x, y, z)=\left(G_{x} x+F_{x x} x^{2}, G_{y} y+F_{y y} y^{2}, G_{z} z+F_{z z} z^{2}\right)$
If $G_{x}=G_{y}=G_{z}=0.001 \mathrm{~T} / \mathrm{m}$ and $F_{x x}=F_{y y}=F_{z z}=0.02 \mathrm{~T} / \mathrm{m}^{2}$, the breadth of the simulated spectrum of Fig. $3 \mathrm{~B}$ is around $20 \mathrm{kHz}$. This leads to a zig-zag trajectory that deviates from the ideal path during the $D 1$ decoding sequence. The $D 2$ scheme allows one to refocus these deviations at $t=2(n-1) T_{d}$ with $n=1,2, \ldots, N_{d}$ (corresponding to the origin of the $x$ axis), leaving significant deviations near $x_{\max }$. With the $D 3$ scheme combined with a delay $\tau=T_{d}$ $/ 2$, the distorted and ideal trajectories intersect halfway at $x_{\max } / 2$ rather than at $x_{0}$, and the errors are attenuated by a factor 2 .

\subsection{Resolution of CE-MRI in homogeneous fields}

As for spatial encoding using chirped pulses [5], the resolution of CE-MRI depends only on the encoding conditions. In Fig. 4, we present numerical simulations of the image of square and rectangular two-dimensional objects obtained with the pulse scheme of Fig. 1A. In Fig. 4A-C, the overall phase $\Phi$ of Eq. (8) was weighted by factors $0.1,1$ and 10 , respectively. This allows one to scale the encoding parameter $\lambda$ while keeping the same field of view (FOV). The three two-dimensional images and central cross-sections through the square object at $x=0$ or $z=0$ illustrate how the resolution depends on $\lambda$. The resolution can be improved by increasing the strength and/or length of the encoding gradients. The decoding gradients affect only the FOV but have no influence on the resolution.

By comparing the images of the rectangular $2 \times 30 \mathrm{~mm}$ object in Fig. $1 \mathrm{C}$ and the square $2 \times 2 \mathrm{~mm}$ object in Fig. $4 \mathrm{~B}$, one observes a difference in resolution, although the encoding parameters were similar. Clearly, the resolution depends on the dimensions of the object. In Figs. 4D-F, we show that the elongation of the object
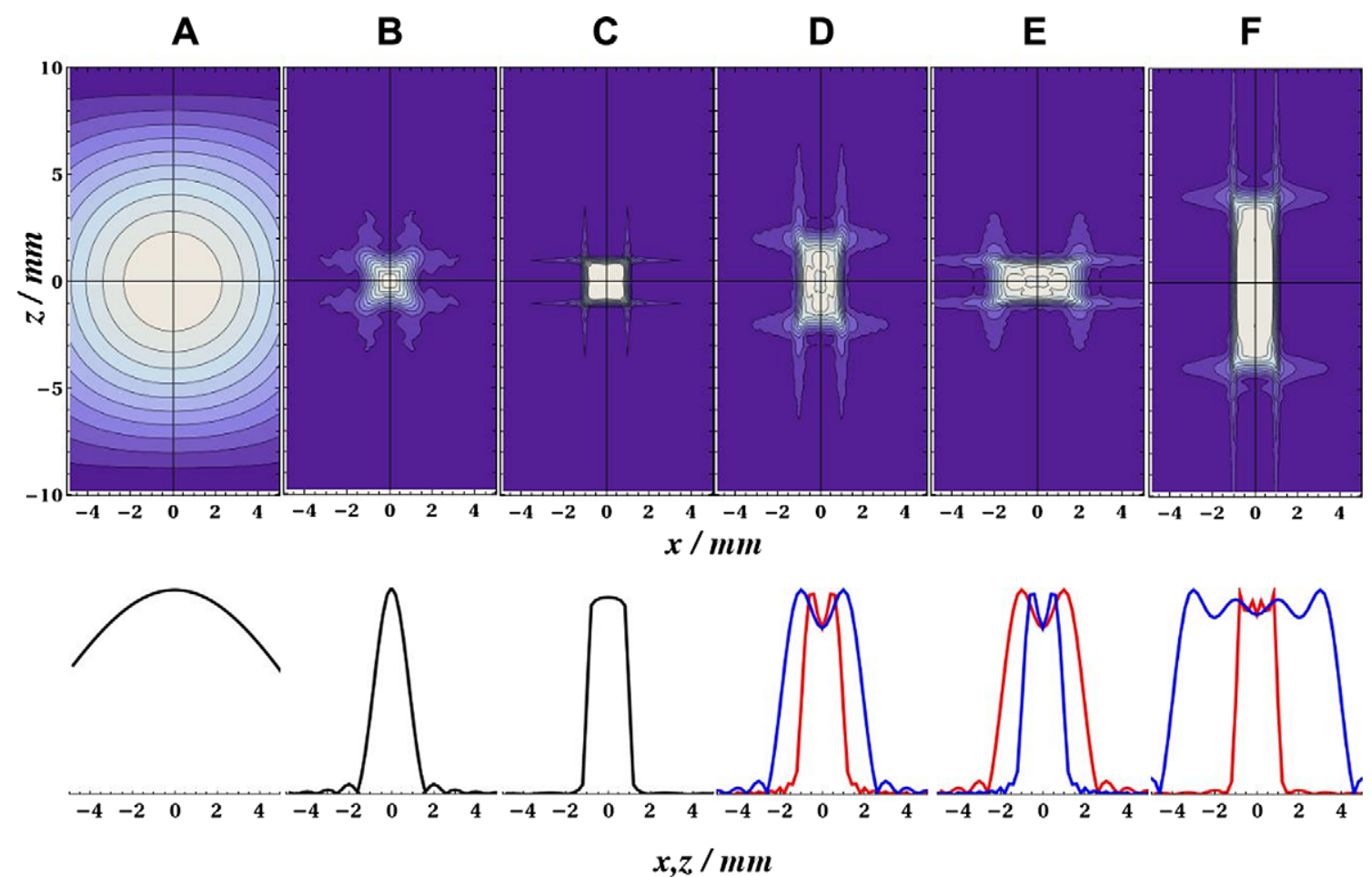

Fig. 4. Effect of encoding parameters and dimensions of the object on the resolution. As in Fig. 1C, numerical simulations of the images of square and rectangular two dimensional objects were obtained with the pulse scheme of Fig. $1 \mathrm{~A}$. The absolute value of the integral of Eq. (4) was calculated at intervals $\Delta x=x_{\max } / 50$ and $\Delta z=z_{\max } / 100$ with the following parameters: $G_{x}^{e}=G_{z}^{e}=0.022 \mathrm{~T} / \mathrm{m}, G_{x}^{d}=0.0241 \mathrm{~T} / \mathrm{m}$ and $G_{z}^{d}=0.3864 \mathrm{~T} / \mathrm{m}, T_{e}=6 \mathrm{~ms}, T_{d}=320 \mu \mathrm{s}, \Delta \omega_{a d} /(2 \pi)=40 \mathrm{kHz}, N_{d}=16$, resulting in $x_{\mathrm{max}}=10 \mathrm{~mm}$ and $z_{\max }=20 \mathrm{~mm}$ (FOV $=10 \times 20 \mathrm{~mm}$ ). From A to C, the overall phase $\Phi$ of Eq. (8) was weighted by factors $0.1,1$ and 10 , respectively. The $2 \mathrm{D}$ images and central crosssections (blue lines for vertical cross-sections at $x=0$, red lines for horizontal cross-sections at $z=0$ ) of a $2 \times 2$ mm square object illustrate the dependence of the resolution on the encoding parameter. Elongation of the object along the $z$ axis to $2 \times 4 \mathrm{~mm}$ (D) or $2 \times 8 \mathrm{~mm}$ (F) or along the $x$ axis to $4 \times 2 \mathrm{~mm}$ (E) increases the resolution in the perpendicular direction. (For interpretation of the references to color in this figure legend, the reader is referred to the web version of this paper.) 

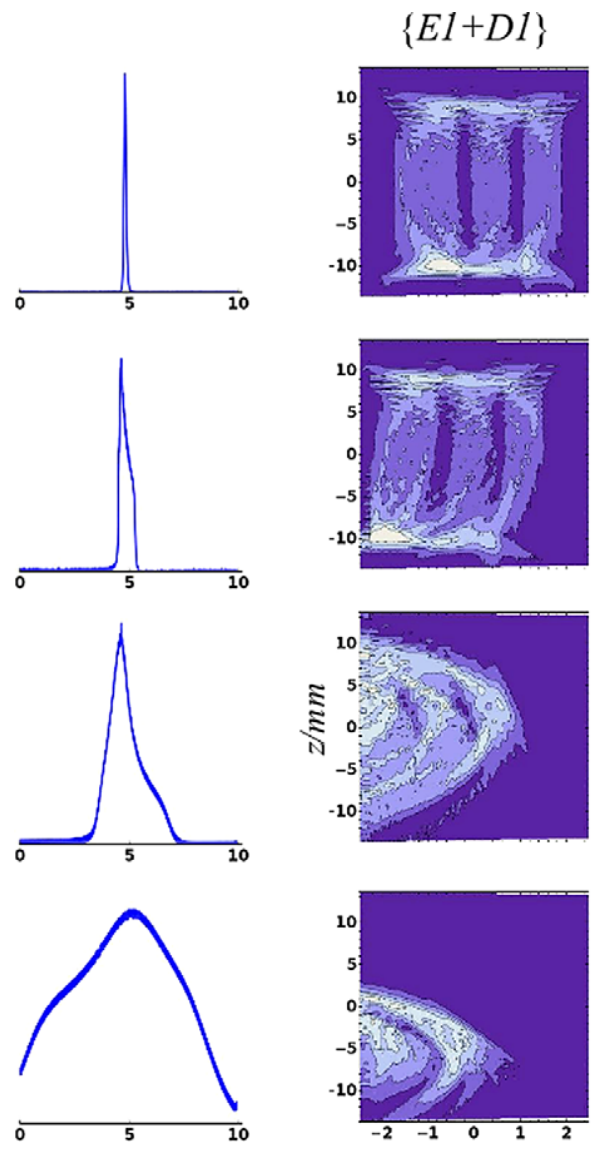

ppm

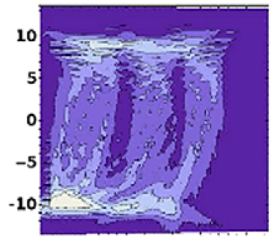

$\{E 1+D 3\}$
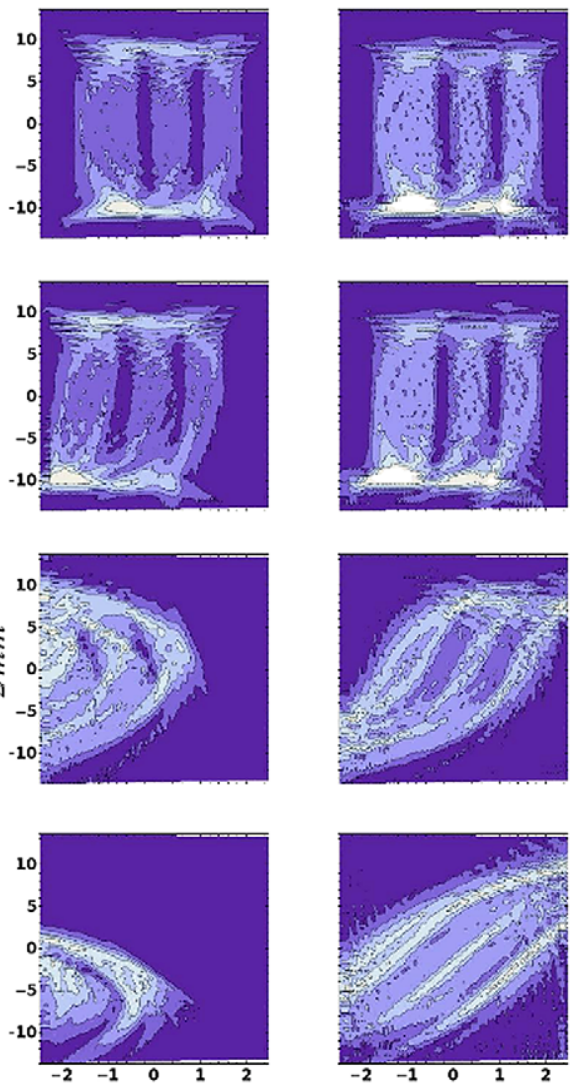

$\{E 1+E 2+D 1\}$
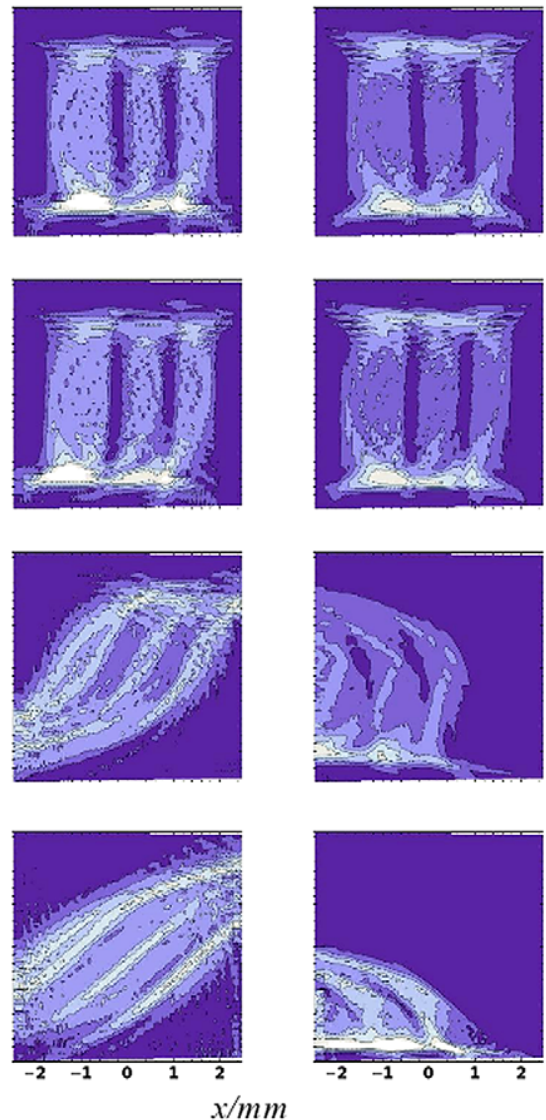
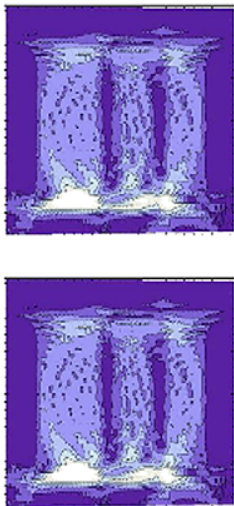

$\{E 1+E 2+D 3\}$
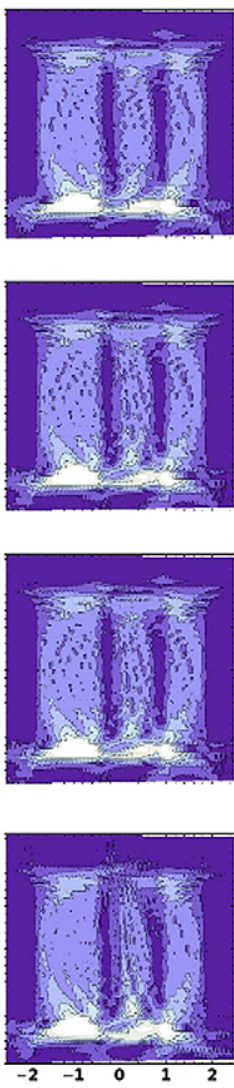

Fig. 5. Comparison of experimental images of a phantom with various combinations of encoding and decoding schemes for an increasingly inhomogeneous static field $\delta B_{0}(x$, $y, z$ ), characterized by the lineshapes of the water resonance in the absence of any pulsed field gradients (left). A glass capillary (0.9 and 1.6 mm inner and outer diameters) was inserted into a tube with a $3.8 \mathrm{~mm}$ inner diameter; the spaces inside the capillary and between the two tubes being filled with water. The experimental conditions were $G_{x}^{e}=0.022 \mathrm{~T} / \mathrm{m}$ (for $E 1$ ) or $G_{x}^{e}=0.011 \mathrm{~T} / \mathrm{m}$ (for $E 1+E 2$ ), $G_{z}^{e}=0.022 \mathrm{~T} / \mathrm{m}, G_{x}^{d}=0.0165 \mathrm{~T} / \mathrm{m}, G_{z}^{d}=0.1925 \mathrm{~T} / \mathrm{m}, T_{e}=6 \mathrm{~ms}, T_{d}=320 \mu \mathrm{s}, \Delta \omega_{a d} /(2 \pi)=40 \mathrm{kHz}, N_{d}=32$. All experiments were performed with a Bruker Avance $600 \mathrm{MHz}(14 \mathrm{~T})$ spectrometer equipped with a triple-gradient TBI probe.

in one dimension increases the resolution in the perpendicular direction. In Fig. 4D and E, the object is elongated either along the $z$ axis $(2 \times 4 \mathrm{~mm})$ or along the $x$ axis $(4 \times 2 \mathrm{~mm})$. This leads to an improvement of the resolution in the $x$ and $z$ dimensions respectively. The figures also illustrate that the dimensions of the FOV have no impact on the resolution. A comparison of the cross-sections in Fig. 4B, D and F (the latter with a $2 \times 8 \mathrm{~mm}$ object) highlights a gain in resolution (sharper transitions near the edges) in the $x$ dimension (red lines) while the resolution in the $z$ dimension (blue lines) remains the same (same slope in transition regions).

\section{Experimental results}

Fig. 5 shows experimental images obtained with a phantom consisting of a glass capillary (0.9 and $1.6 \mathrm{~mm}$ for the inner and outer diameters) inserted in a sample tube $(3.8 \mathrm{~mm}$ inner diameter), where the spaces are filled with water. Various combinations of encoding and decoding schemes were tested from left to right, for increasing static field inhomogeneities $\delta B_{0}(x, y, z)$ from top to bottom, characterized by the lineshapes of the water resonance recorded in the absence of any pulsed field gradients (left margin). The images are increasingly distorted from top to bottom. Used in isolation, the encoding $\{E 1+E 2\}$ schemes or the decoding $D 3$ sequence cannot cope with very large inhomogeneities. Only the combination $\{E 1+E 2+D 3\}$ gives nearly undistorted images in the most inhomogeneous fields. The combination $\{E 1+E 2+D 2\}$ (not shown) gives slightly larger distortions.

Fig. 6 (top half) shows applications of the combined scheme $\{E 1+E 2+D 3\}$ to the same phantom as used in Fig. 5 and (bottom half) to a different phantom consisting of a glass spiral inserted in a sample tube (3.8 $\mathrm{mm}$ inner diameter). This demonstrates how the new method yields 3D images that are virtually indistinguishable when recorded under dramatically different conditions, regardless whether the homogeneity is good or severely perturbed. The geometrical characteristics of the two phantoms can be determined quite accurately even when the inhomogeneity of the static field leads to a water resonance with a breadth of about $3000 \mathrm{~Hz}$.

\section{Conclusions}

The novel cross-encoded MRI schemes, using adiabatic pulses and alternating gradients in two perpendicular dimensions, can remove undesirable effects of inhomogeneous magnetic fields that may result from susceptibility effects and prevent blurring due to slow motion since the images are recorded in a fraction of a second. The zig-zag trajectories in the decoding period can be improved by using refocusing pulses. Magnetic resonance images have been recorded in two and three dimensions that are virtually indistinguishable in spite of dramatically different static field inhomogeneities. 


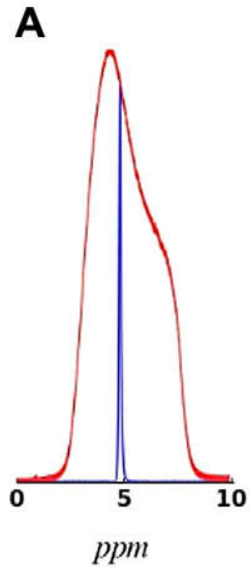

B

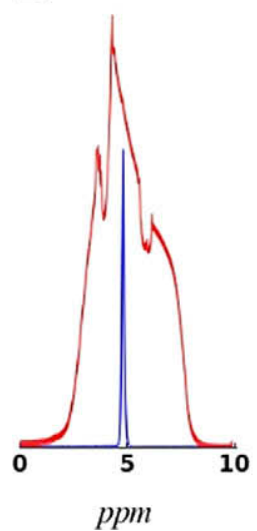

.

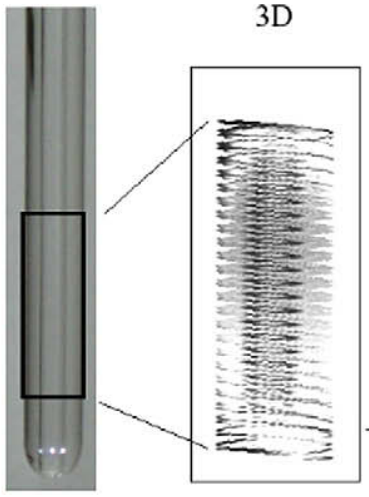

Homogeneous field

$3 \mathrm{D}$

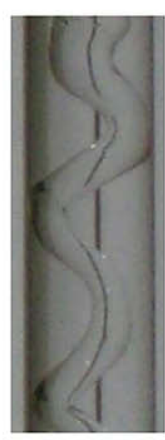

.

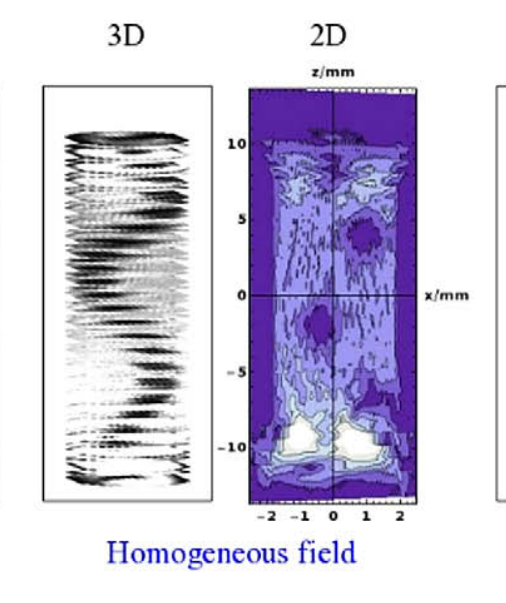

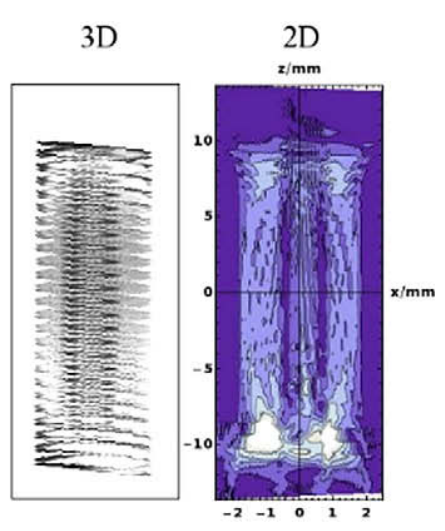

Inhomogeneous field

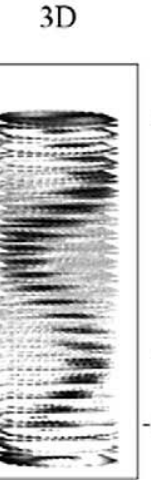

Inhomogeneous field

Fig. 6. Images obtained with the combined scheme $\{E 1+E 2+D 3\}$ of Figs. 2 and 3 with $(A)$ the same glass capillary phantom as in Fig. 5 , and (B) with a glass spiral phantom surrounded by water inserted into a tube with $3.8 \mathrm{~mm}$ inner diameter. Both phantoms were immersed either in homogeneous or inhomogeneous fields characterized by the proton spectra on the left (blue or red lines, respectively). In the $y$-dimension, 32 neighboring slices were recorded with a recovery delay of $10 \mathrm{~s}$ between scans. Photographs of the phantoms (black-and-white) are compared with full 3D images obtained by addition of the 32 planes (grey scale) and with 2D images of the central slices (blue scale). In order to visualize the cross-sections of the phantoms, the different planes of the 3D images are stacked following the experimental zig-zag pattern, like the fold of an accordion. The experimental conditions were like in Fig. $5: G_{x}^{e}=0.011 \mathrm{~T} / \mathrm{m}, G_{z}^{e}=0.022 \mathrm{~T} / \mathrm{m}, G_{x}^{d}=0.0165 \mathrm{~T} / \mathrm{m}, G_{z}^{d}=0.1925 \mathrm{~T} / \mathrm{m}, T_{e}=6 \mathrm{~ms}, T_{d}=320 \mu \mathrm{s}, \Delta \omega_{a d} /(2 \pi)=40 \mathrm{kHz}, N_{d}$ $=32, \tau=T_{d} / 2$. A Bruker Avance $600 \mathrm{MHz}(14 \mathrm{~T})$ spectrometer equipped with a triple-gradient TBI probe was used. (For interpretation of the references to color in this figure legend, the reader is referred to the web version of this paper.)

\section{Acknowledgments}

We thank Laetitia Zuccarelli for manufacturing the helical glass phantom. This work was supported by the European Union (Integrated Infrastucture Initiative, Contract No. RII3-026145, Joint Research Activity JRA1, Contract No. 026145), the Centre National de la Recherche Scientifique (CNRS, France), the Agence Nationale pour la Recherche (ANR, France), the Fonds National de la Recherche Scientifique (FNRS, Switzerland, No. 200020_124694/1) and the Commission pour la Technologie et l'Innovation (CTI, Switzerland, No. 9991.1 PFIW-IW).

\section{References}

[1] P.C. Lauterbur, Image formation by induced local interactions: examples employing nuclear magnetic resonance, Nature 242 (1973) 190-191.

[2] S. Ogawa, T.M. Lee, A.S. Nayak, P. Glynn, Oxygenation-sensitive contrast in magnetic resonance image of rodent brain at high magnetic fields, Magn Reson. Med. 14 (1990) 68-78.
[3] K.K. Kwong et al., Dynamic magnetic resonance imaging of human brain activity during primary sensory stimulation, Proc. Natl. Acad. Sci. USA 89 (1992) 5675-5679.

[4] M. Stehling, R. Turner, P. Mansfield, Echo-planar imaging: magnetic resonance imaging in a fraction of a second, Science 254 (1991) 43-50.

[5] L. Frydman, T. Scherf, A. Lupulescu, The acquisition of multidimensional NMR spectra within a single scan, Proc. Natl. Acad. Sci. USA 99 (2002) 15858-15862.

[6] Y. Shrot, L. Frydman, Spatially encoded NMR and the acquisition of 2D magnetic resonance images within a single scan, J. Magn. Reson. 172 (2005) 179-190.

[7] A. Kumar, D. Welti, R.R. Ernst, NMR Fourier Zeugmatography, J. Magn. Reson. 18 (1975) 69-83.

[8] A. Tal, L. Frydman, Spatial encoding and the single-scan acquisition of high definition MR images in inhomogeneous fields, J. Magn. Reson. 182 (2006) 179-194.

[9] P. Pelupessy, Adiabatic single scan two-dimensional NMR spectroscopy, J. Am. Chem. Soc. 125 (2003) 12345-12350.

[10] M.H. Levitt, Composite pulses, Prog. Nucl. Magn. Reson. Spectrosc. 18 (1986) 61-122.

[11] D.N. Guilfoyle, P. Mansfield, K.J. Packer, Fluid flow measurement in porous media by echo-planar imaging, J. Magn. Reson. 97 (1992) 342-358.

[12] B. Manz, P.S. Chow, L.F. Gladden, Echo-planar imaging of porous media with spatial resolution below 100 m, J. Magn. Reson. 136 (1999) 226-230. 